\title{
DISTRIBUCIÓN DE LOS MACROLÍQUENES CORTICÍCOLAS Y SU RELACIÓN CON LA VEGETACIÓN EN EL PARQUE NATURAL DE LOS ALCORNOCALES (CÁDIZ, S DE ESPAÑA).
}

\author{
Yolanda HERNÁNDEZ GALLEGO, Antonio DÍAZ ESPEJO y Jorge G. ROWE
}

\begin{abstract}
RESUMEN. Distribución de los macroliquenes corticicolas y su relación con la vegetación en el Parque Natural de Los Alcornocales (Cádiz, $S$ de España).Se estudiaron los macrolíquenes corticícolas en distintas unidades de vegetación: alcornocal (Quercus suber L.), quejigal (Quercus canariensis Willd.) y acebuchal (Olea europaea L. var. oleaster DC.); la influencia de la orientación en la composición y número de especies; y como afectaba el perímetro del forófito a la diversidad de macrolíquenes corticícolas. Se observó una clara diferencia en la distribución de los macrolíquenes en el quejigal frente a la del acebuchal y el alcornocal. Sólo existían diferencias entre las orientaciones norte y sur en el acebuchal. También se observó una disminución en la diversidad de los macrolíquenes corticícolas al aumentar el perímetro del forófito en el acebuchal.
\end{abstract}

Palabras clave. Distribución, líquenes corticícolas, Parque Natural Los Alcornocales.

SUMMARY. Distribution of the bark macrolichens and its relationship with the vegetation in the Alcornocales Natural Park (Cádiz, southern Spain). Bark macrolichens were studied in different vegetation communities: evergreen cork oak (Quercus suber L.) woodlands, semideciduous oak (Quercus canariensis Willd.) woodlands and wild olive trees (Olea europaea L. var. oleaster DC.); the influence of lichen orientation on the composition and species number, as well as the effect of the phorophyte perimeter on the bark macrolichens diversity were studied. Semideciduous oak woodlands were found clearly different from the other groups of samples. Differences between north and south orientation in the group of plots of wild olive trees were detected. It was also observed that in the wild olive trees, the macrolichen diversity decreased when the phorophyte perimeter increased.

Keywords. Distribution, bark macrolichens, Los Alcornocales Natural Park

\section{INTRODUCCIÓN}

La importancia de las comunidades de líquenes en los ecosistemas forestales no es comparable a la de otros productores primarios; no suelen constituir un eslabón fundamental en las cadenas tróficas ni contribuyen significativamente a su estructura. Aunque no parecen tener un papel importante, constituyen probablemente el mejor elemento bioindicador de la complejidad y grado de madurez de estos ecosistemas. Además, suministran una gran 
información con respecto a los principales factores ambientales como humedad, temperatura, iluminación, y delatan gran número de microambientes que se configuran en los sistemas maduros.

El estudio de la distribución de los líquenes ha sido abordado desde distintos enfoques, entre ellos: la distribución y sucesión en las ramas (Hilmo, 1994), comunidades de macrolíquenes epífitos sobre Quercus suber en diferentes series de vegetación (Fos et al, 1994), distribución de macrolíquenes sobre el tronco de diferentes especies de forófitos (Kershaw, 1964) y sobre ramas y tronco de la misma especie de forófito (Crespo et al, 1982).

En el Parque Natural (P.N.) de Los Alcornocales (Cádiz) la vegetación de plantas vasculares ha sido ampliamente estudiada (Ojeda, 1995: 37), mientras que los trabajos sobre vegetacion liquénica son escasos (Rowe J.G. y Egea J.M., 1986; 1987a; 1987b; Sequeiros et al, 1986). Como consecuencia, este trabajo es una aproximación a la distribución de los macrolíquenes corticícolas en el P.N. de Los Alcornocales. Los objetivos de nuestro estudio fueron los siguientes:

Determinar si la composición liquénica es diferente en las distintas unidades de vegetación superior.

Estudiar la influencia de la orientación (N/S) en la composición y número de especies.

Determinar la influencia del perímetro del forófito en la diversidad de macrolíquenes corticícolas.

\section{MATERIAL Y MÉTODOS}

\section{Área de estudio.}

Este estudio fue realizado en el P.N. Los Alcornocales. Dicho parque, ocupa buena parte de la franja oriental de la provincia de Cádiz en su limite con la de Málaga, desde el borde sur del P.N. de Grazalema hasta las playas comprendidas entre Tarifa y la Bahía de
Algeciras, incluyendo en su zona nororiental algunas hectáreas de territorio malagueño. Con una extensión total de 170.025 ha. es el tercer P.N. más grande de Andalucía.

El clima es mediterráneo con inviernos frescos y húmedos y veranos cálidos y secos. La precipitación anual total oscila entre 665 $1210 \mathrm{~mm}$, aunque en algunas zonas del Parque se puede llegar a alcanzar los $2000 \mathrm{~mm}$ de media. La temperatura media anual es de $17^{\circ} \mathrm{C}$. Los frecuentes vientos húmedos del este (levante) y la proximidad a la costa suavizan las condiciones climáticas.

Quercus suber ocupa la mayor extensión del área, Quercus canariensis se ve relegado al fondo de los valles, sobre suelos profundos, derivados de areniscas, húmedos y muy humificados. Los brezales densos aparecen en zonas altas de los montes, sobre suelos ácidos derivados de areniscas. Una especie arbórea abundante en el área es Olea europaea, que se asienta sobre suelos calizos, margosos o margoarenosos.

Las unidades de alcornocal y quejigal muestreadas en nuestro estudio, han sido previamente descritas por Ojeda (1995).

\section{Método de muestreo.}

Se ha realizado un muestreo estratificado al azar en las siguientes unidades de vegetación superior: alcornocal, acebuchal y quejigal. Otras unidades descritas en la zona de estudio no se han muestreado, puesto que en ellas no aparece suficiente cobertura de macrolíquenes corticícolas.

En cada una de estas unidades se delimitaron 5 macroparcelas de $20 \times 50 \mathrm{~m}$, eligiendo zonas alejadas de la influencia de la carretera, dentro de las cuales se eligieron aleatoriamente 5 forófitos. En éstos, se distribuyeron dos microparcelas de $10 \times 25 \mathrm{~cm}$, a 1,60 m de altura, una en orientación norte y otra en orientación sur, donde se anotó las presencia de las distintas especies de macrolíquenes. 
Se tomó nota también del perímetro de los forófitos. Se tuvo en cuenta que los individuos elegidos fueran de perímetros similares y de tronco recto, para evitar influencias de otros factores, como cambios en las comunidades debido a la sucesión y en el grado de eutrofización, respectivamente. A la vez, se anotaron las especies de macrolíquenes corticícolas no inventariados en las microparcelas. De esta forma, intentamos recoger la riqueza de especies de cada macroparcela.

Para determinar la influencia del perímetro del tronco en la diversidad de macrolíquenes, se realizó un muestreo específico, eligiendo 30 forófitos de diferentes perímetros $(50-200 \mathrm{~cm})$ en la unidad de acebuchal, todos en una misma zona del área de estudio, para evitar un factor más de variabilidad. Este muestreo sólo fue realizado en el acebuchal, puesto que, en el alcornocal los árboles de mayor perímetro están descorchados y en el quejigal era difícil encontrar un rango amplio de perímetros en una misma zona. En cada uno de ellos se volvieron a distribuir 2 microparcelas de $10 \times 25$ $\mathrm{cm}$ en orientación norte y sur. Estas microparcelas estaban subdivididas en 40 celdillas para poder estimar el porcentaje de cobertura de briófitos y de cada una de las especies de macrolíquenes.

Para la identificación de las especies de líquenes se ha utilizado basicamente Purvis et al (1992) y Clauzade \& Roux (1985). Todas las muestras recolectadas han sido depositadas en el Herbario del Departamento de Biología Vegetal y Ecología de la Universidad de Sevilla (Seccion Farmacia SEVF).

\section{Análisis de datos.}

Con los datos de presencia/ausencia de las especies en cada microparcela se construyó una matriz de frecuencias de especies en cada macroparcela. Esta matriz de datos fue sometida a un análisis de correspondencia (CA) para estudiar la distribución de las macroparcelas en función de su composición florística.

Así mismo, para estudiar la influencia de la orientación se realizó un CA, en el cual, la matriz utilizada estaba constituida por la frecuencia de cada especie al norte y al sur, para las unidades de vegetación estudiadas. Para contrastar los resultados obtenidos en este último análisis se realizó el test de UMann Whitney.

Por último, se usó el coeficiente de correlación de Spearman para estudiar la influencia del perímetro del forófito en la diversidad de macrolíquenes corticícolas.

\section{RESULTADOS Y DISCUSIÓN}

\section{Distribución de macrolíquenes corticícolas.}

En el análisis de correspondencia (tab. 1 y fig.1) se observa una clara separación entre el grupo de parcelas del quejigal y las pertenecientes al alcornocal y el acebuchal, a lo largo del eje 1. Esto es debido, a la presencia de 3 especies exclusivas de nuestras parcelas de quejigal (Lobaria amplissima, $L$. pulmonaria, Parmelia quercina), y también a la mayor frecuencia de las especies Pertusaria albescens, Physcia pulverulenta y Collema furfuraceum en el quejigal respecto al alcornocal y acebuchal, en las que también aparecen.

La causa de ésto es probablemente el diferente microambiente presente en esta unidad. Según Crespo et al (1982), la especie del forófito no es determinante en la

\begin{tabular}{cccc}
\hline & Eje 1 & Eje 2 & Eje 3 \\
\hline Autovalores & 0.58438 & 0.37756 & 0.17638 \\
\% varianza & 33.31 & 21.52 & 10.05 \\
\hline
\end{tabular}

Tabla 1. Resultado del análisis de correspondencia para las macroparcelas. Result of the correspondence analysis for the macroplots. 


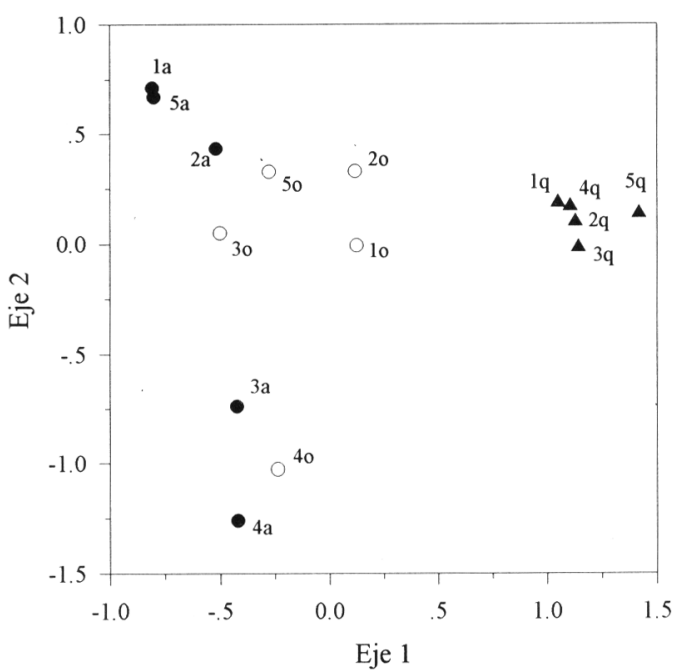

Figura 1. Ordenación de las macroparcelas mediante un análisis de correspondencia (CA). ( $\mathbf{\Delta}$ ) quejigal, (-) alcornocal y $(\mathrm{O})$ acebuchal. Ranking of macroplots by correspondence analysis (CA). quejigal, ( cork oak, and (O) wild olive.

distribución de la flora y vegetación liquénica. La distribución de los líquenes está afectada por factores microclimáticos que son de pequeña importancia para las plantas vasculares (Canters et al., 1991). Ninguna de las macroparcelas presentó una localización dentro del Parque que pudiera provocar la aparición de valores inusualmente bajos en el número de especies, como por ejemplo cercanía a una carretera. En este análisis no se observa una distribución diferente entre las macroparcelas de alcornocal y las de acebuchal. El número de especies comunes en ambas unidades es alto. Por otra parte, esto puede ser debido al bajo porcentaje de varianza que recoge el eje 2 (fig. 1), unido a características intrínsecas de este análisis, que hacen que la información de este eje sea de difícil interpretación.

La diversidad medida como índice de Shannon-Weaver $(\mathrm{H})$ de cada macroparcela fue correlacionada con los valores del eje 1 del CA (fig.2), observándose una correlación

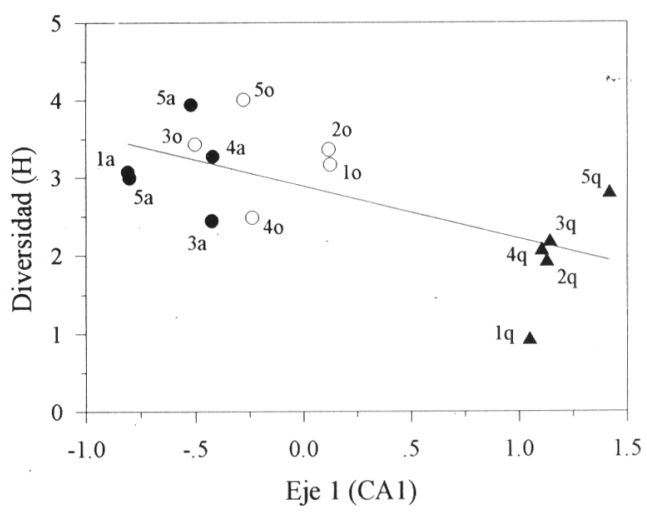

Figura 2. Relación entre la diversidad de las macroparcelas y sus valores en el eje 1 del CA. Los símbolos como en la figura 1 . Se ha representado la recta de regresión que mejor se ajustaba a los puntos $(\mathrm{r}=-0.55 ; \mathrm{p} \leq 0.032)$. Relationship between diversity of the macroplots and their values on axis 1 of the $C A$. The best fit to a straight line of regressions is shown $\left(r_{s}=-0.55 ; p \leq 0.032\right)$.

negativa con el mismo $\left(r_{s}=-0.5536 ; p \leq 0.032\right)$. Este resultado pone de manifiesto la menor diversidad del quejigal frente al acebuchal y al alcornocal.

\section{Influencia de la orientación.}

En cuanto a la influencia de la orientación (tab. 2 y fig.3), el análisis une los puntos norte y sur tanto en el quejigal como en el alcornocal, mientras que en el acebuchal éstos se separan. Para obtener una posible explicación a estos resultados se llevó a cabo un test de U-Mann Whitney contrastando la riqueza de especies (medida como número de especies) en ambas orientaciones para cada unidad, y se analizó si existía un cambio en la diversidad.

Los resultados del test confirman la distribución que aparecía en el CA. No existen diferencias significativas entre el norte y el sur del alcornocal y quejigal, pero sí en el acebuchal $(\mathrm{T}=740 ; \mathrm{p} \leq 0.05 ; \mathrm{n}=25$; muestras no apareadas). La causa podría ser la distinta 


\begin{tabular}{cccc}
\hline & Eje 1 & Eje 2 & Eje 3 \\
\hline Autovalores & 0.52605 & 0.14557 & 0.07267 \\
\% varianza & 66.02 & 18.27 & 9.12 \\
\hline
\end{tabular}

Tabla 2. Resultado del análisis de correspondencia para las orientaciones. Result of correspondence analysis for the orientations.

exposición (luz, humedad) a la que se ven sometidos los macrolíquenes en cada unidad de vegetación. El alcornocal y quejigal presentan una mayor cobertura arbórea, son bosques más cerrados, y por tanto, los líquenes están menos expuestos que en el acebuchal, que es una unidad más abierta, lo cual permite que se pongan de manifiesto las diferencias N/ $\mathrm{S}$. En el estudio de pequeños territorios donde las fluctuaciones térmicas sean pequeñas entre unos puntos y otros, la clave en la distribución de las comunidades liquénicas corresponde al ombroclima, así como al factor iluminación (Crespo et al, 1982).

Se calculó el índice de diversidad de Shannon-Weaver en cada unidad para ambas orientaciones (tab. 3), y los resultados apoyaron los obtenidos con los otros análisis. Sólo se detecta diferencia en la diversidad N/S en el acebuchal.

Además, se observó para cada unidad que un $65-70 \%$ de las especies son comunes en ambas orientaciones y que son muy pocos los indicios a favor de la existencia de especies con afinidad por una orientación concreta.

La figura 2 también aporta más información al primer CA (fig. 1). El quejigal se separa claramente en ambos. Sin embargo, según este segundo CA (fig. 3), parecen existir diferencias entre las unidades de acebuchal y de alcornocal. En estos resultados puede influir la síntesis de información para construir la matriz. Como se muestra en la tabla 4, las diferencias no estriban en la identidad de las especies, sino en un cambio en su frecuencia.

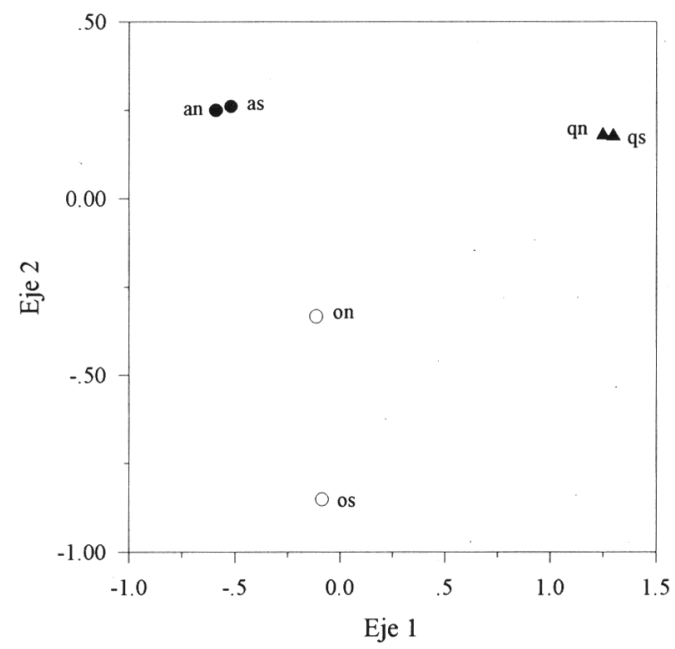

Figura 3. Ordenación de los distintos tipos de vegetación mediante un $\mathrm{CA}$ y en base a su composición liquénica y su orientación $(\mathrm{n}=$ norte, $\mathrm{s}=$ sur). Símbolos como en la figura 1. Ranking of the different types of vegetation by $C A$, based on lichen composition and orientation $(n=n o r t h$, $s=$ south). Symbols as figure 1 .

\section{Influencia del perímetro.}

En la figura 4 a perteneciente a la cara norte, podemos observar que existe una correlación significativa y negativa entre el índice de Shannon-Weaver para las microparcelas estudiadas y el perímetro del forófito. También se estudió la correlación entre el perímetro y la cobertura de briófitos (fig. 4b). En este caso, la cobertura aumenta al

\begin{tabular}{lcc}
\hline Unidad & Orientación & H \\
\hline \multirow{2}{*}{ Quejigal } & Norte & 2.79757 \\
& Sur & 2.60796 \\
Alcornocal & Norte & 4.93489 \\
& Sur & 4.81080 \\
Acebuchal & Norte & 4.82138 \\
& Sur & 3.95762 \\
\hline
\end{tabular}

Tabla 3. Índices de Shannon-Weaver para los líquenes de cada unidad de vegetación en función de su orientación. Shanon-Weaver indices for the lichens of each plant unit depending on orientation. 


\begin{tabular}{|c|c|c|c|}
\hline ESPECIES & Quejigal & Alcornocal & Acebuchal \\
\hline Collema furfuraceum (Arnold) Du Rietz & 40 & 0 & 20 \\
\hline Evernia prunastri (L.) Ach. & 0 & 18 & 18 \\
\hline * Lepraria aeruginosa sensu Ozenda et Clauz. & 0 & 0 & 0 \\
\hline Lobaria amplissima (Scop.) Forss. & 8 & 0 & 0 \\
\hline Lobaria pulmonaria Hoffm. & 8 & 0 & 0 \\
\hline * Nephroma laevigatum Ach. & 0 & 0 & 0 \\
\hline Parmelia borreri (Sm.) Turn. & 2 & 28 & 34 \\
\hline Parmelia caperata (L.) Ach. & 0 & 64 & 24 \\
\hline Parmelia glabratula ssp.fuliginosa (Fr. ex Duby) Laund & d. 0 & 18 & 2 \\
\hline Parmelia olivacea (L.) Ach. & 4 & 6 & 0 \\
\hline Parmelia perlata (Huds.) Vain. & 2 & 22 & 2 \\
\hline Parmelia quercina (Willd.) Vain. & 2 & 0 & 0 \\
\hline Parmelia sulcata Tayl. & 0 & 4 & 0 \\
\hline Parmelia tiliacea (Hoffm.) Ach. & 6 & 52 & 14 \\
\hline Parmeliella plumbea (Lightf.) Vain. & 6 & 2 & 0 \\
\hline Platismatia glauca (L.) W. Culb. et C. Culb. & 4 & 18 & 22 \\
\hline Pertusaria albescens (Huds.) Choisy et Werner & 66 & 20 & 34 \\
\hline Pertusaria amara (Ach.) Nyl. & 0 & 14 & 4 \\
\hline Pertusaria flavida (DC.) Laund. & 2 & 58 & 12 \\
\hline Physcia ascendens (Fr.) Oliv. & 0 & 4 & 14 \\
\hline Physcia semipinnata (Gmel.) Moberg. & 0 & 2 & 2 \\
\hline Physconia venusta (Ach.) Poelt & 20 & 2 & 2 \\
\hline Physconia enteroxantha Nyl.) Poelt & 0 & 40 & 18 \\
\hline Physconia pulverulacea Moberg & 64 & 2 & 10 \\
\hline Ramalina farinacea (L.) Ach. & 14 & 34 & 18 \\
\hline Teloschistes chrysophthalmus (L.) Th. Fr. & 0 & 6 & 2 \\
\hline Usnea sp. & 0 & 28 & 6 \\
\hline Xanthoria parietina (L.) Th. Fr. & 0 & 0 & 8 \\
\hline
\end{tabular}

(*) Especies sólo encontradas en los análisis cualitativos de las macroparcelas

Tabla 4. Frecuencias de las especies de líquenes para cada unidad. Frecuency of the species of lichens for each unit.

aumentar el perímetro.

La causa de la disminución de la diversidad de los líquenes podría ser la competencia que se establece con briófitos en el norte. Al realizar la correlación entre $\mathrm{H}$ de líquenes y cobertura briófitos ésta no es significativa (fig. 4c), por lo que no se puede afirmar que la disminución en $\mathrm{H}$ esté relacionada con el aumento de la cobertura de briófitos.
En la cara sur, el perímetro del forófito y la diversidad de los líquenes presentan correlación negativa (fig. 4d), al igual que en la cara norte. En la cara sur no aparecen briófitos, por lo cual estos resultados apoyan a los de la correlación anterior e impiden afirmar que la disminución de la diversidad con el perímetro en la cara norte sea debido exclusivamente a la competencia con briófitos. 
a)

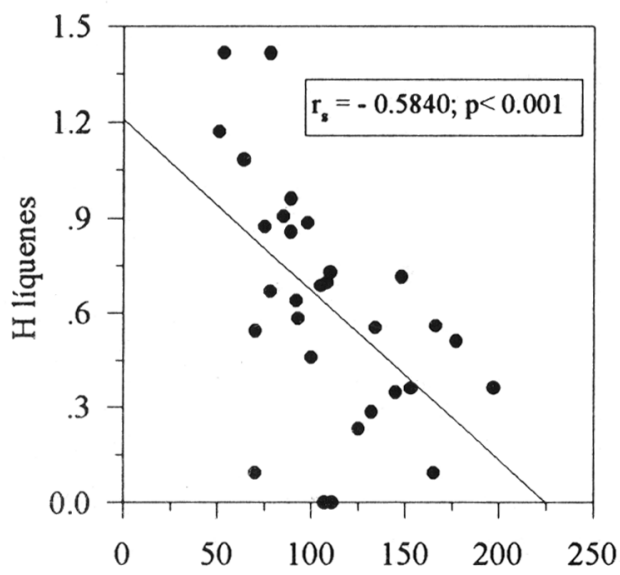

Perímetro forófito

c)

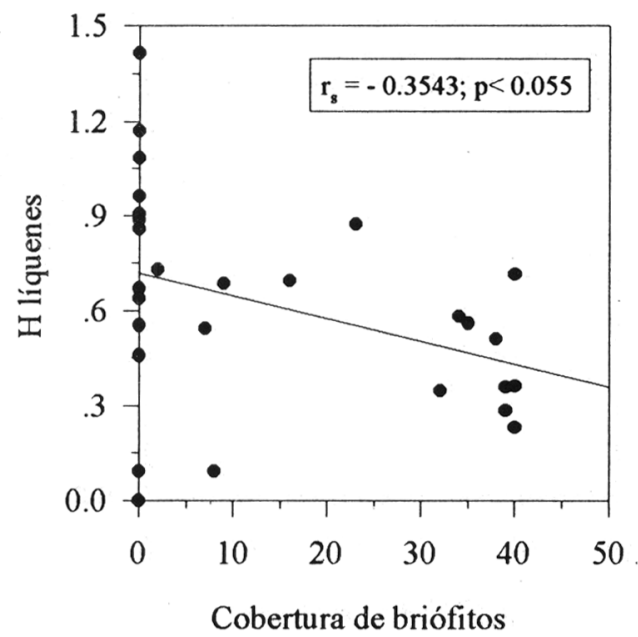

b)

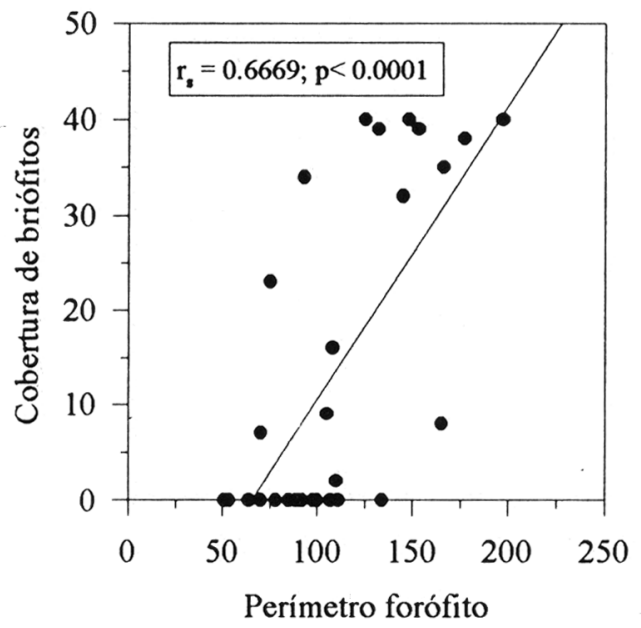

d)

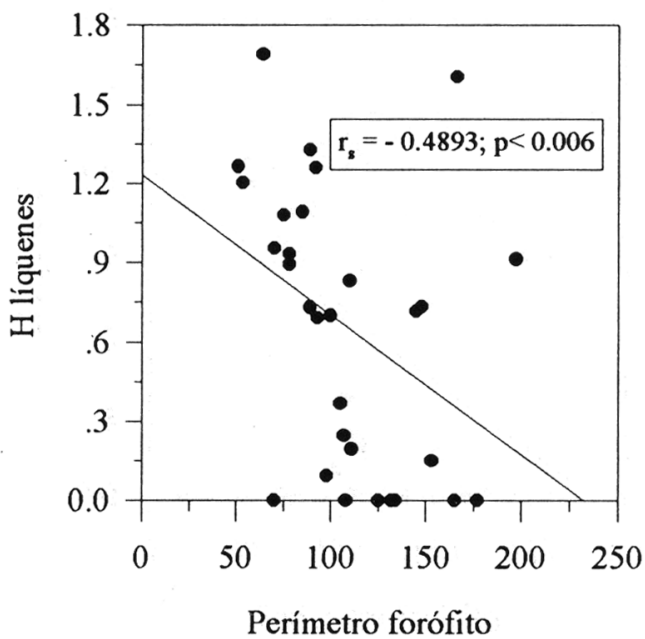

Figura 4. Correlaciones entre: a) diversidad de líquenes en la cara norte y perímetro del forófito, b) cobertura de briófitos y perímetro del forófito, c) diversidad de líquenes y cobertura de briófitos y d) diversidad de líquenes y perímetro del forófito en la cara sur. Al igual que en el caso anterior se han dibujado las rectas de regresión que mejor se ajustaban a los puntos, en todos los casos $n=30$. Correlations between: a) diversity of lichens on the north face and phorophyte perimeter; $b$ ) cover of Bryophytes and phorophyte perimeter; c) diversity of lichens and cover of Bryophytes; and d) diversity of lichens and phorophyte perimeter on the south face. As in the former case, the best-fitting straight lines of regression are shown. In all cases, $n=30$. 
AGRADECIMIENTOS. Agradecemos la ayuda prestada por el Dr. D. Juan Arroyo, Profesor Titular del Departamento de Biología Vegetal y Ecología de la Facultad de Biología, Universidad de Sevilla.

\section{BIBLIOGRAFÍA}

CANTERS, K.J., H. SCHÖLLER, S. OTT \& H.M. JAHNS -1991- Microclimatic influences on lichen distribution and community development. Lichenologist 23 (3): 237-252.

CLAUZADE, G. \& C. ROUX -1985- Likenoj de Okcidenta Europo. Bull. Soc. Bot. Centre-Ouest 7.

CRESPO, A., E. BARRENO y L.G. SANCHO 1982- Esbozo de la flora y vegetación liquénicas de algunas localidades de los valles del Tambre y Ulla (Coruña, España). 2 $2^{\mathbf{a}}$ Jornadas Fitosociología. Santiago de Compostela.

FOS, S., V. ATIENZA, M.J. SANZ, V. CALATAYUD y E. BARRENO -1994Macrolíquenes epifitos sobre Quercus suber L. en los alcornocales del centro y sur de Portugal. Studia Botanica 13: 139-145.

HILMO, O. -1994- Distribution and succession of epiphytic lichens on Picea abies branches in a boreal forest, Central Norway. Lichenologist 26 (2): 149-169.

KERSHAW, K.A. -1964- Preliminary observations on the distribution and ecology of epiphytic lichens in Wales. Lichenologist 2: 263-276.

OJEDA, F. -1995-Ecología, biogeografía y diversidad de los brezales del Estrecho de Gibraltar (Sur de España, Norte de Marruecos). Tesis Doctoral. Sevilla.

PURVIS, O.W., B.J. COPPINS, D.L. HAWKSWORTH, P.W. JAMES \& D.M. MOORE -1992- The lichen flora of Great Britain and Ireland. Natural History Museum Publications.

ROWE, J.G. y J.M. EGEA -1986- Líquenes silicícolas de las sierras costeras occidentales de la Comarca de Algeciras (Prov. Cádiz, Sur de España). Acta Bot. Malacitana 11:55-74.

ROWE, J.G. y J.M. EGEA -1987a- Pertusarietum mammoso-gallicae y Lecanactino-Dirinetum sorediatae, dos nuevas asociaciones liquénicas rupícolas. Anales Jard. Bot. Madrid 44 (1): 119-129.
ROWE, J.G. y J.M. EGEA -1987b- Algunos líquenes silicícolas de la Sierra del Aljibe (Provincia de Cádiz, Sur de España). Studia Botanica 6: 8994.

SEQUEIROS, M.V., J.G. ROWE y S. SILVESTRE -1986- Contribución al conocimiento de los líquenes epífitos de la provincia de Cádiz, Sur de España, I. Studia Botanica 5: 85-104.

Aceptado para su publicación en Octubre de 1998

Dirección de los autores. Y. Hernández Gallego y A. Díaz Espejo: Dept. Biología Vegetal y Ecología. Facultad de Biología. Universidad de Sevilla. J.G. Rowe: Dept. Biología Vegetal y Ecología. Facultad de Farmacia. Universidad de Sevilla. 